\title{
A Panel of miRNAs as Prognostic Markers for African-American Patients with Triple Negative Breast Cancer
}

\section{Safaa Turkistani}

Howard University

\section{Bruna M Sugita}

Research Institute Pelé Pequeno Príncipe

\section{Paolo Fadda}

The Ohio State University

\section{Rafael Marchi}

Research Institute Pelé Pequeno Príncipe

\section{Ali Afsari}

Howard University

\section{Tammey Naab}

Howard University

\section{Victor Apprey}

Howard University

Robert L Copeland Jr

Howard University

Michael Campbell

Howard University

Luciane R Cavalli ( $\sim$ Irc@georgetown.edu )

Research Institute Pelé Pequeno Príncipe

\section{Yasmine Kanaan}

Howard University

\section{Research Article}

Keywords: microRNA, triple-negative breast cancer, African-American, prognosis, tumor size, lymph node

Posted Date: January 27th, 2021

DOl: https://doi.org/10.21203/rs.3.rs-151939/v1 
License: (c) (i) This work is licensed under a Creative Commons Attribution 4.0 International License. Read Full License

Version of Record: A version of this preprint was published at BMC Cancer on July 27th, 2021. See the published version at https://doi.org/10.1186/s12885-021-08573-2. 


\section{Abstract}

Background: To investigate the global expression profile of miRNAs, their impact on cellular signaling pathways and their association with poor prognostic parameters in African-American (AA) patients with triple negative breast cancer (TNBC).

Methods: Twenty-five samples of AA TNBC patients were profiled for global miRNA expression and stratified considering three clinical-pathological parameters: tumor size, lymph node $(L N)$, and recurrence (REC) status. Differential miRNA expression analysis was performed for each parameter, and their discriminatory power was determined by Receiver Operating Characteristic (ROC) curve analysis. KMplotter was assessed to determine the association of the miRNAs with survival, and functional enrichment analysis to determine the main affected pathways and miRNA/mRNA target interactions.

Results: A panel of eight, 23 and 27 miRNAs were associated with tumor size, LN, and REC status, respectively. Combined ROC analysis of two (miR-2117, and miR-378c), seven (let-7f-5p, miR-1255b-5p, miR-1268b, miR-200c-3p, miR-520d, miR-527, and miR-518a-5p), and three (miR-1200, miR-1249-3p, and miR-1271-3p) miRNAs showed a robust discriminatory power based on tumor size (AUC=0.917), LN $(A U C=0.945)$ and REC (AUC=0.981) status, respectively. Enrichment pathway analysis revealed their involvement in proteoglycans and glycan, and cancer-associated pathways. Eight miRNAs with deregulated expressions in patients with large tumor size, positive LN metastasis and recurrence were significantly associated with lower survival rates.

Conclusions: Altogether, our data indicate that miRNA deregulated expression is a relevant biological factor that can be associated with the poor prognosis in TNBC of AA patients, by conferring to their TNBC cells aggressive phenotypes that are reflected in the clinical characteristics evaluated in this study.

\section{Background}

Breast cancer is one of the most frequent types of female cancer worldwide and one of the main causes of death in women [1]. Several new target therapies are currently in development with significant potential to reduce mortality in patients with varied breast cancer subtypes by significantly increasing their overall survival [2]. However, for the triple negative breast cancer (TNBC), there are still few approved targeted therapies. Inhibitors of the programmed death-ligand 1 (PDL1) have been recently approved for the treatment of unresectable locally advanced or metastatic TNBC patients. This targeted therapy is, however, only beneficial for patients with PDL1 positive tumor expression $[3,4]$.

TNBC is considered one of the most aggressive subtypes of breast cancer, with high rates of progression and poor prognosis [5]. Comprehensive molecular-based studies have been extensively performed to identify new biomarkers that can be used for diagnosis, prognosis, and more efficient treatment regimens for this tumor subtype. One class of biomarkers that has emerged with promising therapeutic potential is the non-coding microRNAs (miRNAs) [6, 7]. With the technological advances in the nanoparticle delivery 
systems, this class of biomarkers has been shown to be effective in inhibiting tumor progression and metastasis in several tumor models, including TNBC [8-10].

MiRNAs are conserved endogenous small RNA molecules that can regulate a range of developmental and physiological processes in the cells, in a tissue-specific manner [11]. MiRNAs expression alterations are associated with the development of pathological processes and chronic diseases [12]. In cancer, miRNAs play a critical role in tumor initiation and progression, through their regulatory role in the expression of gene targets involved in multiple signaling pathways, such as cell proliferation, differentiation and cell death [13-17]. In TNBC, a number of miRNAs with deregulated expression have been identified and associated with aggressive cancer phenotypes, including larger tumor sizes, early tumor recurrence, lymph node, and metastatic invasion, and lower survival rates [18].

Several studies have shown that miRNAs present variable expression patterns according to race and/or ethnic groups [19-23]. However, few of them were conducted in patients' tumor samples. We have previously shown in genomically characterized African-American (AA) and Latina patients with TNBC, differential patterns of tumor miRNA expression when compared to TNBC patients of European descent $[24,25]$. These profiles were shown to be associated with distinct signaling pathways, commonly linked to poor prognosis and shorter survival. Although there are a limited number of studies evaluating the somatic miRNA expression in tumors of genomically ancestral characterized populations, this data supports the relevance of miRNAs as biological contributors to cancer disparities.

In this study, the main objective was to characterize the global patterns of miRNA expression, and their impact on cellular signaling pathways in the TNBC tissue specimens of AA patients. The miRNA patterns were also evaluated according to three selected clinical-pathological parameters: tumor size, lymph node (LN) and recurrence (REC) status.

\section{Material And Methods}

\subsection{Samples Collection, Clinical and Demographic Information}

Formalin-fixed paraffin-embedded tissue blocks (FFPE) of 25 TNBC patients were collected from the surgical pathology archives of Howard University Hospital, Washington DC. Clinical and histopathological data were obtained for the patients in a codified manner according to the approved institutional IRB protocols and included age at diagnosis, histologic subtype, tumor size, Nottingham score tumor stage, lymph node (LN), recurrence (REC), and distant metastasis status.

Clinical and histopathological data of the variables above were retrieved for all the cases analyzed. Most of the patients $(81.8 \%)$ were diagnosed with infiltrating ductal carcinoma, with a mean age at diagnosis of $55.27 \pm 9.96$ years old (range 32 to 78 years old). All tumors, but one (score 7), were classified as high grade in the Nottingham Histologic Score and Grade (score 8/9). A wide variability in tumor size was 
observed, with tumors presenting their largest dimension between 1.1 and $22 \mathrm{~cm}$ in size (average $5.86 \pm$ $5.17 \mathrm{~cm})$. Ten patients $(62.5 \%)$ presented tumors smaller than $5 \mathrm{~cm}$, while six patients $(37.5 \%)$ presented tumors larger than $5 \mathrm{~cm}$. $L N$ involvement $(\mathrm{LN}+)$ was present in ten patients $(47.6 \%)$ affecting one or more LNs while eleven patients (53.4\%) were LN negative (LN-). Sixty-eight percent of the patients became disease-free after treatment and had no recurrence, while $32 \%$ relapsed (with local and/or distant metastasis) after the selected treatment regimen or never reached a disease-free condition since diagnosis (Table S1).

The classification of the TNBC phenotype was confirmed by immunohistochemistry analysis using ER, $\mathrm{PR}$, and HER2 receptors markers, following current guidelines [26, 27].

The AA race information of the patients was initially obtained from self-reported medical records and later confirmed by genotyping analysis for a subset of the patients. The genotyping was performed using the SNP chip Illumina Infinium QC Array (Illumina Inc., CA), which contains 15,949 markers, including, 3,000 ancestral informative markers (AIMs). The genotype calling was performed as we previously described [24, 25], using the GenomeStudio Software v. 2011.1. Genotypes from the mitochondrial genome and on sex chromosomes were excluded, as well as genotypes with call rate $<98 \%$. The remaining autosomal genotypes (8,687 in total) were integrated with the variant calls from $\geq 1,900$ individuals originating from 21 diverse populations in the 1000 Genomes Project. To explore population structure among individuals, Principal Component Analysis (PCA) was conducted on the genome-wide autosomal loci. First, a genetic relationship matrix between pairs of individuals (GRM files) was generated with the GCTA software [28]. Then, using the GRM files as input, the PCA method implemented in GCTA was applied. In these analyses, the default setting $(n=20)$ was accepted, which outputted the first 20 eigenvectors and all of the eigenvalues. Lastly, the top two Principal Components, PC1, and PC2 were plotted using RStudio [29]. PC1 (accounting for $69.1 \%$ of the extracted variation) broadly distinguished individuals of African descent from non-Africans, suggesting some genetic distance between African and non-African populations consistent with previous studies of autosomal loci [30, 31] (Figure S1).

\subsection{RNA Isolation and Global miRNA Expression Analysis}

The FFPE tumor specimens were evaluated by a pathologist for the presence of at least $80 \%$ of tumor cells. The selected tumor areas were microdissected from unstained 10 $\mu \mathrm{m}$ FFPE tissue sections and total RNA was isolated using TRIzol (Invitrogen Carlsbad, CA, USA) after deparaffinization with Xylene solution. RNA concentration and quality were tested by measuring $260 / 280$ and $260 / 230$ ratios using Nanodrop 2000 spectrophotometer (Willington, DE, USA).

MiRNA expression analysis was performed using Nanostring nCounter Human v3a miRNA Expression Assay (Seattle, WA, USA) that contains human probes derived from miRBase version 22 (http://www.mirbase.org) targeting 827 human miRNAs, six positive controls, eight negative controls, three ligation positive controls, three ligation negative controls, five internal reference genes $(A C T B, B 2 M$, GAPDH, RPL19, and RPLO), and five spike-in controls (ath-miR-159a, cel-miR-248, cel-miR-254, osa-miR- 
414, and osa-miR-442). Raw data were pre-processed using Nanostring's Counter RCC collector and normalized using Nanostring nSolver 4.0 software (Background subtraction: the geometric mean of Negative Controls; Technical normalization: the geometric mean of Positive Controls; Codeset content normalization: all genes geometric mean). Normalized data was Log2 transformed and analyzed using the MultiExperiment Viewer software (MeV 4.9.0), GraphPad Prism 8.3.0 and IBM SPSS Statistics 25.

\subsection{Differential miRNA Expression Analysis Among the TNBC Clinical Groups}

The tumor samples profiled for miRNA were stratified in different clinical groups according to three selected clinical-pathological parameters: tumor size ( $\geq 5 \mathrm{~cm}$ or $<5 \mathrm{~cm}$ ), LN status [positive ( $L N+)$ or negative (LN-) and REC status (positive (REC+) or negative (REC-)]. Differential miRNA expression analysis was performed for each of these parameters, resulting in three distinct miRNA panels (Welch based $t$-Test, $\mathrm{p} \leq 0.05$ ). Log2 Fold Change (Log2FC) was calculated for each differentially expressed miRNA. Unsupervised and Supervised Hierarchical Clustering analysis was performed using Pearson's correlation coefficient and average linkage and visualized as heatmaps with a 3-color scheme blue-blackyellow representing values below the median-median-above median, respectively.

\subsection{Receiver Operating Characteristic (ROC) Curve Analysis}

The discriminatory power of the differentially expressed miRNAs between the different clinical groups was tested by constructing the ROC curve and calculating the Area Under the Curve (AUC). Sensitivity was plotted against specificity (\%) for the binary classifiers ( $\geq 5 \mathrm{~cm} v s<5 \mathrm{~cm}, \mathrm{LN}+v s \mathrm{LN}-$, and REC + vs REC-). An AUC of $1.0(100 \%)$ denotes perfect discrimination by the miRNA, whereas an AUC of $0.5(50 \%)$ denotes a complete lack of discrimination by the miRNA. AUCs and $95 \%$ corresponding confidence intervals (Cl) were calculated for each miRNA using GraphPad Prism v 8.3.0 and IBM SPSS Statistics 25.

Multiple binary logistic regression analysis was used to determine the effect of the panel of miRNAs in discriminating the samples according to their tumor size, LN, and REC status. The most robust miRNA panel combination was also determined for each clinical parameter studied.

\subsection{Kaplan Meier (KM) Plot Analysis}

The miRpower KM Plotter Tool [32] was used to calculate hazard ratios, confidence intervals, and log-rank $P$ values for the miRNAs that were shown to present a high discriminatory power in each clinical group evaluated. This analysis was performed in relation to survival in the aggregated breast cancer clinical studies extracted from The Cancer Genome Atlas (TCGA) and Molecular Taxonomy of Breast Cancer International Consortium (METABRIC) databases (extraction based on TNBC cases in general, and TNBC cases with known LN status).

\subsection{Functional Enrichment Pathways Analysis}

The Diana miRPath v.3.0 [33] was used to identify the top signaling pathways that were affected by the significant differentially expressed miRNAs, which is based on the online tool Kyoto Encyclopedia of 
Genes and Genomes (KEGG) annotation. For these analyses, micro-T-CDS (v5.0) was used to identify the predicted gene targets regulated by the selected miRNAs, applying the p-value of 0.05 and a microT threshold of 0.8 .

The miRpathDB v.2.0 [34] was used to determine the potential influence of the selected miRNAs in the Integrated Breast Cancer pathway (WP1984) from Wikipathways [35], considering experimentally validated and predicted target genes. This pathway presents relevant proteins and their interactions in breast cancer and has been widely used in miRNA prediction analyses [36, 37].

\subsection{In Silico Functional Analysis}

The databases miRTarBase [38] and miRnet [39] were used to determine interactions between the selected miRNAs and target genes validated based on strong (reporter assays, Western Blot and qPCR) and less strong experimental assays (microarray, NGS, pSilac). MirTargetLink Human online software was used to visualize miRNA-mRNA interaction networks [40].

The STRING v.11 database [41] was used to verify protein-protein interactions (PPI) between the validated target genes of each group, applying the minimum interaction score of 0.9 (highest confidence). Cytoscape v.3.8.0 [42] was used to construct molecular interaction networks of selected miRNAs and target genes.

\section{Results}

\subsection{Differential miRNA Expression Analysis Among the TNBC Clinical Groups}

A total of 25 cases were successfully profiled for the expression of global miRNAs. The analysis of miRNAs expression was performed separately for each of the three selected clinical groups (tumor size, LN and REC status) (Table 1, Fig. 1). In the analysis of the cases based on tumor size, eight miRNAs were observed differentially expressed between the large $(\geq 5 \mathrm{~cm})$ and small $(<5 \mathrm{~cm})$ tumors, from which two miRNAs were upregulated and six downregulated in the large tumor size cases $(t-T e s t p<0.05$, Fig. 1a). In the LN group analysis, 23 miRNAs were observed differentially expressed between the LN + and LNcases, seven upregulated and 14 downregulated in the $L N+$ cases ( $t$-Test $p<0.05$, Fig. 1b). Finally, 27 miRNAs were observed differentially expressed between REC + and REC- cases, seven upregulated and 19 downregulated in the REC + cases ( $t$-Test, $p<0.05$, Fig. 1c). 
Table 1

Log2FC, p-value, AUC and corresponding $95 \% \mathrm{Cl}$ for each differentially expressed miRNA observed in the tumor size, LN and REC status groups.

\begin{tabular}{|c|c|c|c|c|}
\hline Tumor size $(\geq 5 \mathrm{~cm} /<5 \mathrm{~cm})$ & Log2FC & p-value & AUC & $95 \% \mathrm{Cl}$ \\
\hline miR-1281 & -0.87487 & 0.0479 & 0.6833 & 0.4101 to 0.9565 \\
\hline miR-2117 & -1.51124 & 0.0026 & 0.875 & 0.6826 to 1.0000 \\
\hline miR-378c & 1.24370 & 0.0457 & 0.8083 & 0.5528 to 1.0000 \\
\hline miR-452-5p & -0.81783 & 0.0329 & 0.7000 & 0.4376 to 0.9624 \\
\hline miR-5196-5p & -1.27827 & 0.0132 & 0.7500 & 0.5050 to 0.9950 \\
\hline miR-519c-3p & -1.27906 & 0.0187 & 0.8000 & 0.5804 to 1.0000 \\
\hline miR-617 & -0.82711 & 0.0402 & 0.7167 & 0.4536 to 0.9797 \\
\hline miR-934 & 1.610418 & 0.0193 & 0.8333 & 0.6295 to 1.0000 \\
\hline LN status & Log2FC & p-value & AUC & $95 \% \mathrm{Cl}$ \\
\hline let-7f-5p & 1.308153 & 0.0345 & 0.8091 & 0.6141 to 1.000 \\
\hline miR-1253 & -2.23171 & 0.0436 & 0.7909 & 0.5984 to 0.9834 \\
\hline miR-1255b-5p & -1.43984 & 0.0298 & 0.8091 & 0.6201 to 0.9981 \\
\hline miR-1268a & -0.9362 & 0.0441 & 0.7682 & 0.5632 to 0.9732 \\
\hline miR-1268b & 0.7279 & 0.0084 & 0.8636 & 0.6882 to 1.000 \\
\hline miR-128-1-5p & -1.49312 & 0.0254 & 0.7818 & 0.5838 to 0.9799 \\
\hline miR-133a-5p & 1.097722 & 0.0369 & 0.6727 & 0.4348 to 0.9106 \\
\hline miR-200c-3p & 2.602164 & 0.0187 & 0.8182 & 0.6375 to 0.9988 \\
\hline miR-301a-5p & 0.583352 & 0.0212 & 0.8091 & 0.6170 to 1.000 \\
\hline miR-3074-3p & -0.85799 & 0.0301 & 0.7273 & 0.4976 to 0.9570 \\
\hline miR-323b-3p & -1.30263 & 0.0324 & 0.7636 & 0.5366 to 0.9907 \\
\hline miR-367-3p & -0.653 & 0.0438 & 0.7545 & 0.5444 to 0.9647 \\
\hline miR-378f & 1.143995 & 0.0356 & 0.8000 & 0.6058 to 0.9942 \\
\hline miR-513b-5p & -1.04378 & 0.0115 & 0.7864 & 0.5790 to 0.9938 \\
\hline$m i R-520 d-5 p+m i R-527+m i R-518 a-5 p$ & -1.53273 & 0.0161 & 0.8364 & 0.6645 to 1.000 \\
\hline$m i R-520 f-3 p$ & -1.7368 & 0.0355 & 0.7909 & 0.5852 to 0.9966 \\
\hline miR-548I & -0.59929 & 0.0187 & 0.7909 & 0.5936 to 0.9882 \\
\hline
\end{tabular}




\begin{tabular}{|c|c|c|c|c|}
\hline Tumor size $(\geq 5 \mathrm{~cm} /<5 \mathrm{~cm})$ & Log2FC & p-value & AUC & $95 \% \mathrm{Cl}$ \\
\hline miR-548q & -1.98804 & 0.0105 & 0.7727 & 0.5600 to 0.9855 \\
\hline miR-580-3p & 1.266989 & 0.0094 & 0.8045 & 0.5962 to 1.000 \\
\hline miR-595 & -1.17619 & 0.0070 & 0.8182 & 0.6281 to 1.000 \\
\hline miR-873-5p & -0.86919 & 0.0434 & 0.7045 & 0.4769 to 0.9321 \\
\hline REC status & Log2FC & p-value & AUC & $95 \% \mathrm{Cl}$ \\
\hline miR-10a-5p & -0.86382 & 0.022 & 0.7905 & 0.5801 to 1.000 \\
\hline miR-1200 & -1.55119 & 0.0004 & 0.8571 & 0.7004 to 1.000 \\
\hline miR-1249-3p & -1.54646 & 0.0211 & 0.8286 & 0.6493 to 1.000 \\
\hline miR-1271-3p & 0.962012 & 0.0108 & 0.9143 & 0.7968 to 1.000 \\
\hline miR-130a-3p & -1.45528 & 0.0144 & 0.7810 & 0.5850 to 0.9769 \\
\hline miR-184 & -2.01337 & 0.0005 & 0.8857 & 0.7442 to 1.000 \\
\hline miR-18a-5p & -1.2983 & 0.0226 & 0.7667 & 0.5637 to 0.9696 \\
\hline miR-197-3p & 1.067639 & 0.0412 & 0.7714 & 0.5689 to 0.9740 \\
\hline miR-208a-3p & -0.94776 & 0.0372 & 0.7333 & 0.5162 to 0.9505 \\
\hline miR-362-5p & -1.31405 & 0.0486 & 0.7619 & 0.5401 to 0.9837 \\
\hline miR-376a-2-5p & -1.07002 & 0.0055 & 0.7048 & 0.4876 to 0.9220 \\
\hline miR-411-5p & 1.185286 & 0.0084 & 0.7810 & 0.5876 to 0.9743 \\
\hline miR-449b-5p & -1.76057 & 0.0003 & 0.8571 & 0.6997 to 1.000 \\
\hline miR-4536-3p & -1.25336 & 0.0292 & 0.8000 & 0.6039 to 0.9961 \\
\hline miR-491-5p & -0.97535 & 0.0373 & 0.6952 & 0.4761 to 0.9144 \\
\hline miR-495-5p & -1.18756 & 0.0291 & 0.7238 & 0.5092 to 0.9385 \\
\hline miR-5001-5p & -1.12657 & 0.0165 & 0.7905 & 0.5968 to 0.9841 \\
\hline miR-517c-3p + miR-519a-3p & -1.31722 & 0.0364 & 0.6952 & 0.4759 to 0.9146 \\
\hline$m i R-518 f-3 p$ & 1.233145 & 0.003 & 0.8286 & 0.6538 to 1.000 \\
\hline miR-542-3p & -1.47531 & 0.0253 & 0.8095 & 0.6106 to 1.000 \\
\hline miR-548n & 1.427446 & 0.0044 & 0.8619 & 0.6799 to 1.000 \\
\hline miR-587 & 1.032396 & 0.0112 & 0.7714 & 0.5633 to 0.9796 \\
\hline miR-593-3p & 1.172516 & 0.0446 & 0.8190 & 0.6257 to 1.000 \\
\hline
\end{tabular}




\begin{tabular}{|lllll|}
\hline Tumor size $(\geq 5 \mathrm{~cm} /<5 \mathrm{~cm})$ & Log2FC & p-value & AUC & $95 \% \mathrm{Cl}$ \\
\hline miR-595 & -1.00655 & 0.0136 & 0.8381 & 0.6600 to 1.000 \\
\hline miR-891a-5p & -1.23041 & 0.0142 & 0.7619 & 0.5570 to 0.9668 \\
\hline miR-99b-5p & -1.3409 & 0.0267 & 0.8286 & 0.6574 to 0.9998 \\
\hline
\end{tabular}

MiRNA differential expression analysis was also performed based on the four combined LN and REC status groups: LN+/REC+, LN-/REC+, LN+/REC- and LN-/REC- (ANOVA, $p<0.01$ ). Twenty-two miRNAs were found differentially expressed among these groups (Fig. 2). The comparison of the differentially expressed miRNAs among the LN, REC and combined LN/REC status groups resulted in eight miRNAs: miR-10a-5p, miR-1271-3p, miR-184, miR-18a-5p, miR-411-5p, and miR-542-3p present in the REC and LN/REC comparisons, and miR-1253 present in LN and LN/REC comparisons, and miR-595 present in all the three comparisons.

\subsection{ROC Analysis}

ROC analysis was performed for each miRNA in each of the clinical groups evaluated. The analysis of the eight miRNAs observed differentially expressed according to tumor size, resulted in AUC values from 0.683 (miR-1281) to 0.875 (miR-2117). Four miRNAs presented AUC higher than 0.800 and were used to determine the most robust miRNA panel for classifying the samples based on tumor size $(\geq 5 \mathrm{~cm}$ or < $5 \mathrm{~cm})$. As a result, the combination of miR-2117 and miR-378c showed the highest discriminatory power with an AUC value of 0.917(Fig. 3a).

Eleven out of the 23 differentially expressed miRNAs according to the $L N$ status presented AUC $>0.800$, and the combination of let-7f-5p, miR-1255b-5p, miR-1268b, miR-200c-3p, miR-520d-5p + miR-527 + miR518a-5p expression levels presented the highest discriminatory power (AUC $=0.945$; Fig. $3 b$ ).

Finally, the ROC analysis of miRNAs differentially expressed according to REC status, showed an AUC ranging from 0.695 (miR-491-5p and miR-517c-3p + miR-519a-3p) to 0.914 (miR-1271-3p). Twelve miRNAs presented AUC values higher than 0.800 , and a combined ROC analysis indicates that the most robust miRNA panel consisted of miR-1200, miR-1249-3p and miR-1271-3p (AUC = 0.981; Fig. 3c). The individual AUC values for all the miRNAs differentially expressed per clinical group and their corresponding $95 \% \mathrm{Cl}$ are presented in Table 1 .

\subsection{Association of the Target miRNA Genes with Survival Using KM Plot Database}

The miRNAs that were shown to present a high discriminatory power (AUC > 0.8) in each clinical group analyzed were queried in relation to survival in TNBC samples of the TCGA and METABRIC cohorts, using the KM Plot database. In the tumor size group, only one (miR-2117) out of four miRNAs, was observed in association with survival (Fig. 4a). Lower expression of this miRNA, as we observed in the group of patients with larger tumor sizes, was significantly associated with shorter survival. In the LN group, eight 
(miR-1225b, miR-301a, miR-378f, miR-518a, miR-520d, miR-527, miR-580; miR-595) out of 12 miRNAs were associated with survival, some of which specifically associated with LN- (miR-1225b, miR-301a, miR-520d, and miR-580) or LN+ (miR-378f, miR-527) patients (Fig. 4b). From these miRNAs, all, except miR-580a and miR-595, presented in the KM Plot, the same direction of the expression levels as we observed in our cases in the LN + group. Finally, in the group of patients based on REC status, four out of 11 miRNAs, presented the association with survival in the TNBC samples of the TCGA and METABRIC cohorts. In this group, only miR-4536, which we observed with lower expression in the REC + group of patients, was associated with shorter survival (Fig. 4c).

\subsection{Functional Enrichment Pathways}

The potentially affected KEGG pathways by the differentially expressed miRNAs were determined for each of the clinical groups evaluated. The top 10 KEGG pathways ranked by $p$-value for each analysis are shown in Table 2. 
Table 2

Top 10 KEGG pathways potentially affected by the differentially expressed miRNAs in each clinical group evaluated: tumor size, LN, and REC status (ranked by p-value).

\begin{tabular}{|c|c|c|c|c|}
\hline$\#$ & KEGG pathways & p-value & $\begin{array}{l}\# \\
\text { genes }\end{array}$ & $\begin{array}{l}\# \\
\text { miRNAs }\end{array}$ \\
\hline & \multicolumn{4}{|l|}{ Tumor size $(\geq 5 \mathrm{~cm},<5 \mathrm{~cm})$} \\
\hline 1 & Ubiquitin mediated proteolysis & $1.46 \mathrm{E}-05$ & 40 & 7 \\
\hline 2 & Circadian rhythm & 0.002970639 & 14 & 7 \\
\hline 3 & Proteoglycans in cancer & 0.002970639 & 42 & 7 \\
\hline 4 & Renal cell carcinoma & 0.004487894 & 18 & 7 \\
\hline 5 & $\begin{array}{l}\text { Signaling pathways regulating pluripotency of stem } \\
\text { cells }\end{array}$ & 0.004646529 & 33 & 6 \\
\hline 6 & Endocytosis & 0.004646529 & 44 & 8 \\
\hline 7 & Glioma & 0.007370446 & 17 & 7 \\
\hline 8 & Oocyte meiosis & 0.015388458 & 25 & 7 \\
\hline 9 & RNA degradation & 0.022814164 & 21 & 6 \\
\hline \multirow[t]{2}{*}{10} & Prolactin signaling pathway & 0.022814164 & 17 & 6 \\
\hline & \multicolumn{4}{|l|}{ LN status } \\
\hline 1 & Mucin type O-Glycan biosynthesis & $3.42 \mathrm{E}-10$ & 14 & 8 \\
\hline 2 & Proteoglycans in cancer & $6.78 \mathrm{E}-10$ & 32 & 9 \\
\hline 3 & $\begin{array}{l}\text { Signaling pathways regulating pluripotency of stem } \\
\text { cells }\end{array}$ & $3.68 \mathrm{E}-07$ & 55 & 9 \\
\hline 4 & TGF-beta signaling pathway & 3.17E-06 & 68 & 8 \\
\hline 5 & $\begin{array}{l}\text { Glycosaminoglycan biosynthesis - heparan } \\
\text { sulfate/heparin }\end{array}$ & $5.72 \mathrm{E}-06$ & 38 & 7 \\
\hline 6 & Hippo signaling pathway & $6.54 \mathrm{E}-06$ & 2 & 2 \\
\hline 7 & FoxO signaling pathway & $6.94 \mathrm{E}-06$ & 49 & 8 \\
\hline 8 & Circadian rhythm & 2.90E-05 & 52 & 9 \\
\hline 9 & ErB signaling pathway & 3.54E-05 & 44 & 9 \\
\hline \multirow[t]{2}{*}{10} & Phosphatidylinositol signaling system & $6.34 \mathrm{E}-05$ & 25 & 7 \\
\hline & \multicolumn{4}{|l|}{ REC status } \\
\hline 1 & Prion diseases & 1.07E-05 & 10 & 8 \\
\hline
\end{tabular}




\begin{tabular}{|c|c|c|c|c|}
\hline \# & KEGG pathways & p-value & $\begin{array}{l}\# \\
\text { genes }\end{array}$ & $\begin{array}{l}\text { \# } \\
\text { miRNAs }\end{array}$ \\
\hline 2 & Proteoglycans in cancer & 1.07E-05 & 91 & 20 \\
\hline 3 & Mucin type 0-Glycan biosynthesis & $1.34 \mathrm{E}-05$ & 15 & 12 \\
\hline 4 & Cell adhesion molecules (CAMs) & $1.84 \mathrm{E}-05$ & 65 & 18 \\
\hline 5 & TGF-beta signaling pathway & $3.45 \mathrm{E}-05$ & 40 & 16 \\
\hline 6 & Axon guidance & $3.45 \mathrm{E}-05$ & 65 & 16 \\
\hline 7 & Oxytocin signaling pathway & 0.001164 & 76 & 18 \\
\hline 8 & N-Glycan biosynthesis & 0.003947 & 21 & 15 \\
\hline 9 & Thyroid hormone signaling pathway & 0.003947 & 53 & 19 \\
\hline 10 & $\begin{array}{l}\text { Signaling pathways regulating pluripotency of stem } \\
\text { cells }\end{array}$ & 0.004389 & 65 & 20 \\
\hline
\end{tabular}

The analysis of differentially expressed miRNAs, in the tumor size group, resulted in 21 pathways, including ubiquitin-mediated proteolysis (hsa04120), proteoglycans in cancer (hsa05205), and signaling pathways regulating pluripotency of stem cells (hsa04550). In the LN group, 51 KEGG pathways potentially affected by these miRNAs were identified, including mucin type-0-glycan biosynthesis (hsa00512), TGF-beta signaling pathway (hsa04350), hippo signaling pathway (hsa04390), and ErbB signaling pathway (hsa04012). Finally, considering the REC status of the patients, $41 \mathrm{KEGG}$ pathways were identified potentially affected by these miRNAs, including proteoglycans in cancer (hsa05205), cell adhesion molecules (CAMs) (hsa04514) and N-Glycan biosynthesis (hsa00510).

Considering all the miRNAs selected from the three comparisons among the three clinical groups, 15 miRNAs were found to be involved in the Integrated Breast Cancer Pathway, potentially targeting 133 genes, some of them targeted by more than one miRNA (Table S2).

\subsection{In Silico Functional Analysis and miRNA/mRNA target networks}

To further elucidate the potential biological impact of each differentially expressed miRNA in the clinical groups evaluated, experimentally validated interactions between miRNA and their target genes were assessed (only strong evidence of interaction was considered for this analysis). Thirty-three of the 58 selected miRNAs (2/8 for the tumor size group, 13/23 for $L N$ status and 18/27 for REC status) presented interactions experimentally validated, regulating a total of 295 target genes (Table S3). When considering only miRNAs with high power to discriminate among the cases in each clinical group (based on ROC analysis), a total of 13 miRNAs were observed (one for the tumor size group, seven for LN status and five for REC status) (Table 3). 
Table 3

MiRNAs and corresponding target genes identified in each clinical group evaluated (only miRNAs with high discriminatory power (AUC $\geq 0.8$ ) were included).

\section{Clinical miRNAs Target Genes groups}

$\begin{array}{lll}\text { Tumor } & \text { miR- } & \text { HIF1A, ABCG2, ELAVL1, TIMP2, PTEN, CDKN1A } \\ \text { size } & 519 \mathrm{c}-3 \mathrm{p} & \\ (\geq 5 & \\ \mathrm{cm} /<5 & \\ \mathrm{~cm}) & \end{array}$

LN let-7f- KLK10, KLK6, PRDM1, IL3, CYP19A1, COPS8, GPS1, CCND1, COPS6, MYH9, status $\quad 5 \mathrm{p} \quad$ SOCS3, ELF4, CCL7, AGO1, IL6, POSTN

miR- $\quad$ TUBB3, BMI1, GEMIN2, BAP1, ZEB2, ZEB1, FN1, ZFPM2, PTPN13, RNF2, 200c-3p RCOR3, BRD7, ACVR2B, MSN, NTRK2, ERRFI1, CCNE2, XIAP, BCL2, TIMP2, FBLN5, VEGFA, NCAM1, IKBKB, FLT1, KLF9, TBK1, PMAIP1, NTF3, LPAR1, EDNRA, RHOA, KLHL20, PTPRD, ELMO2, ERBIN, WDR37, VAC14, TCF7L 1, RASSF2, HOXB5, RIN2, KLF11, SEPT7, SHC1, MYB, ETS1, DUSP1, USP25, EFNA1, RND3, DNMT3A, DNMT3B, SP1, CFL2, CDH11, SEC23A, KDR, HFE, DLC1, ATRX, ZNF217, BTC, ZFPM1, PIN1, KRAS, NOTCH1, GATA4, SUZ12, ROCK2, UBQLN1, E2F3, MALAT1, CDK2, PRKCZ, NOS3, SIRT1, FOXO1, PDCD10, ADAM12, PTEN, LEPR, CRKL, MYLK, SH3PXD2A, DNAJC3, JAZF1, RPS6KB1, SLC1A2

$\begin{array}{ll}\text { miR- } & \text { PTEN, BTG1, NDRG2 } \\ 301 \mathrm{a}- & \\ 5 \mathrm{p} & \\ \text { miR- } & \text { PPIB } \\ 520 \mathrm{~d}- & \\ 5 \mathrm{p} & \\ \text { miR- } & \text { MCL 1, PIK3C2A, CCL2 } \\ 518 \mathrm{a}- & \\ 5 \mathrm{p} & \end{array}$

miR- $\quad$ TWIST1

$580-3 p$

miR- $\quad$ PARD6A

595

REC miR- AKT2, INPPL1, NFATC2, SOX7, AGO2, MYC, BCL2, EZR, SND1, GAS1, ZFPM2,

status $184 \quad P D G F B, P L P P 3, A K T 1, B I N 3, P R K C B, P P P 1 R 13 L, T N F A I P 2, P K M$

miR- $\quad$ SIRT1, CCNE2, MET, GMNN, HDAC1, CDC25A, CDK6, MYCN, NEAT1

$449 \mathrm{~b}-$

$5 p$

miR- $\quad$ BIRC5, ILK, MTDH, PIM1, AKT1, BMP7, RPS23, ANGPT2, OTUB1, IGFBP1, CTTN, 542-3p PIK3R7, FZD7

miR- $\quad C D C 274, P R O P 1$

593-3p 


\section{Clinical miRNAs Target Genes}

groups

miR-

PARD6A

595

Protein-protein interactions (PPI) were evaluated considering the selected validated miRNA target genes for each clinical group and used to construct a miRNA/mRNA network (Fig. 5). For the tumor size, one network was generated based on the six target genes of miR-519c-3p (Table 3, Fig. 5a); considering their involvement in biological process (GO), four of these targets were reported as related to negative regulation of growth (PTEN, CDKN1A, HIF1A), of mitotic cell cycle (PTEN, CDKN1A, TIMP2), and of vascular smooth muscle cell proliferation (PTEN, CDKN1A).

PPI analysis for the LN group was generated considering the 113 experimentally validated target genes of seven miRNAs (Table 3). Considering PPI with the highest confidence (interaction score $>0.9000$ ) and removing nodes without connections, a network was generated with 68 target genes of five miRNAs (Fig. 5b).

For the REC comparison, 41 experimentally validated target genes of five miRNAs (Table 3) were used for the PPI analysis. The miRNA/mRNA network was constructed considering the same settings as the LN network (interaction score $>0.900$, nodes without connection were hidden), resulting in a network with 21 genes and four miRNAs (Fig. 5c).

Finally, considering the eight miRNAs common to LN/REC, LN and REC comparisons and the miRTarbase data (Fig. 6), a network was generated with 89 genes experimentally validated as a target of the 8 selected miRNAs. Most of them were validated through weak evidence of interaction, however strong evidence of interaction was also reported for miR-10a and PTEN, miR-184 and BCL2 and EZR, miR-18a-5p and EZR, PTEN, TGFBR2, CDK19, PIAS3, and BCL2, and miR-542-3p and BIRC5.

To determine the interference of all differentially expressed miRNAs obtained in our analysis in the process related to breast tumorigenesis, a network was constructed considering only experimentally validated target genes presented in The Integrated Breast Cancer pathway (Table 4, Figure S2). In this network, several key oncogenes and tumor suppressor genes were found to be targeted by at least one of the differentially expressed miRNAs in our clinical group comparisons, indicating the importance of these miRNAs in breast tumor initiation and progression, affecting cell proliferation, migration and invasion capacity, and response to treatment. 
Table 4

MiRNAs and experimentally validated target genes involved in the Integrated Breast Cancer Pathway (Wikipathways) and their involvement in the clinical groups of this study.

\begin{tabular}{|c|c|c|}
\hline miRNAs & Experimentally validated targets & $\begin{array}{l}\text { Clinical } \\
\text { groups }\end{array}$ \\
\hline miR-1268a & CDC25B, MAPK1, RAP1A & LN status \\
\hline miR-1268b & CDC25B, MAPK1, RAP1A & LN status \\
\hline $\begin{array}{l}\operatorname{miR}-130 a- \\
3 p\end{array}$ & ESR1, MYC, PTEN, SMAD4, TGFBR2 & REC status \\
\hline miR-184 & $A K T 1, B C L 2, M Y C$ & REC status \\
\hline miR-18a-5p & $\begin{array}{l}\text { ATM, BCL2, CCND1, ESR1, PTEN, RAP1A, SMAD2, SMAD4, TFGBR2, } \\
\text { TP53 }\end{array}$ & REC status \\
\hline miR-18b-5p & ESR1, MDM2, SMAD2 & REC status \\
\hline $\begin{array}{l}\text { miR-200c- } \\
5 p\end{array}$ & CDH1, MDM2, PTEN & LN status \\
\hline miR-449-5p & CDC25A, FOSL 1, HDAC1, SIRT1, SMAD4, TGFBR2 & REC status \\
\hline miR-452-5p & BMPR2, IRS1, KRAS, SMAD4 & Tumor size \\
\hline $\begin{array}{l}\operatorname{miR}-519 a- \\
3 p\end{array}$ & PTEN, RB1 & REC status \\
\hline miR-99b-5p & CHEK1, MTOR, SP1 & REC status \\
\hline
\end{tabular}

\section{Discussion}

In TNBC several miRNAs were observed with deregulated expression presenting with major roles in cancer progression $[43,44]$. Most of the studies reporting on the miRNA expression in TNBC do not focus on minority groups or biological disparities based on the race of the patients. In fact, a search on PubMed database using the words "African American" OR "African women" OR "biological disparity" AND microRNA OR microRNAs OR miRNA OR miRNAs resulted in 83 studies related to AA women and microRNAs with only six of them with TNBC patients, which included the previous study of our group [24].

In this study, we report on the global miRNA profiling of genomically ancestral characterized AA patients with TNBC stratified in three clinical groups of patients based on tumor size, LN metastasis and breast cancer recurrence status.

Tumor size is one of the most important prognostic determinants for breast cancer [45]. Larger tumors can be a result of late diagnosis, high proliferation rate, lack of treatment, or poor response to neoadjuvant treatment. In this study, the average tumor size in the non-treated TNBC patients was $5.86 \pm$ $5.17 \mathrm{~cm}$; six of these patients were diagnosed with tumors larger than $5 \mathrm{~cm}$, being categorized as at least Stage IIB (no regional LN metastasis and no clinical or radiological evidence of distant metastasis), 
which as expected, presents lower 5-year survival rates when compared to patients with smaller tumors [46]. Eight miRNAs were found differentially expressed between the groups of patients based on tumor size, among them, the miR-2117, miR-378c, miR519c-3p and miR-934 presented high power (AUC $\geq 0.8$ ) to discriminate the patients in this group. Among these miRNAs, the expression of miR-2117 was observed in colorectal cancer inversely correlated with the expression levels of the target gene TGFBR 1 [47]. This gene is found overexpressed in breast cancer [48] and is involved in the MAPK-Signaling pathway [47]. Downregulation of miR-378c was observed in head and neck squamous cell carcinoma (HNSCC) and appeared as one of the most important prognostic variables for HNSCC [49]. However, in our study we observed lower levels of miR-519c-3p from patients diagnosed with larger tumors $(>5 \mathrm{~cm})$. Increased levels of miR-519c-3p were found to promote tumor growth and proliferation in hepatocellular carcinoma cells, by targeting the BTG3 gene [50]. Increased expression of miR-934 was previously reported on TNBC samples compared to ER + tumors, however, its expression level was not associated with tumor size [51].

Lymph node (LN) status is still one of the strongest prognostic factors in breast cancer [52]. In this group, 23 miRNAs were found differentially expressed between the $\mathrm{LN}+$ and $\mathrm{LN}$ - patients, among them, miR1253 , miR548I and mIR-873-5p, indicating that these miRNAs might be involved in conferring the invasion ability to the tumor cells. A combination of seven of these miRNAs (let-7f-5p, miR-1255b-5p, miR-1268b, miR-200c-3p and miR-520d-5p + miR-527 + miR-518a-5p), presented a more robust discriminatory power (AUC > 0.9). Interestingly, altered expression levels of miR-1253 and miR-548I were reported to interfere in the migration and invasion capacity of non-small cell lung cancer (NSCLC). Altered expression of miR$548 \mathrm{I}$ and miR-1253 were negatively associated with LN metastasis in NSCLC, by targeting the AKT1 and WNT5A genes, respectively $[53,54]$. In our study, both of these miRNAs were observed with lower expression levels in the LN + group, indicating as in NSCLC, a similar tumor suppressor activity in breast cancer. The long non-coding RNA DCST1-AS1 was reported to act as a "sponge" by binding miR-873-5p, resulting in the upregulation of other target genes such as IGF2BP1, MYC, LEF1, and CDD4 and conferring the increase of cell proliferation and metastasis capacity in TNBC cells [55]. The lower expression levels of this miRNA in the LN + group of our study could be among other mechanisms, a consequence of high levels of this IncRNA.

In relation to recurrence (REC), 27 miRNAs were observed differentially expressed between REC + and REC- groups, with 12 presenting an AUC value higher than 0.8; a combined analysis of three of these miRNAs (miR-1200, miR-1249-3p, and miR-1271-3p), presented a robust power (AUC >0.9) in discriminating the patients based on REC status. Deregulated expression levels of miR-1249-3p in breast cancer cells were associated with interference of IncRNA MIF-AS1 [56]. This IncRNA acts as a sponge resulting in lower expression levels of miR-1249-3p which impedes its interaction with the HOXB8 target gene. In vitro upregulation of miR-1249-3p resulted in suppression of proliferation and migration activity and reversion of the Epithelial Mesenchymal Transition (EMT) progress, indicating a tumor suppressor role of this miRNA in breast tumors, and corroborating with our findings that showed lower expression levels of miR-1249-3p in the REC + group. In gliomas and osteosarcomas, it was observed that the downregulation of miR-1200, led to the up-regulation of the HOXB2 gene, which resulted in the increase of 
proliferation and invasion capacity of the tumor cells $[57,58]$. These data indicate a tumor suppressor activity of miR-1200, that as miR-1249-3p, presented lower expression levels in the REC + group. Finally, for miR-1271, to our knowledge, there is no reported deregulation of its expression in tumor cells.

TNBC patients can show satisfactory response to chemotherapy, especially in the neo-adjuvant setting [59-61]. However early recurrence is more frequent in this breast cancer subytpe when compared to others, which usually occurs within the first 3 years after diagnosis [5, 62]. Chemotherapy adherence and uptake have also been shown to differ among patients' racial/ethnic groups [63-66]. In TNBC, a previous study conducted by our group [67], showed however that although a substantial number of TNBC patients failed to receive and/or complete chemotherapy, AA patients presented a higher chemotherapy uptake than White patients. Interestingly, and aware of the limitation of the sample size of this present study, seven patients presented recurrence after treatment while fifteen patients were disease-free. Considering both LN and REC status, 22 miRNAs were found differentially expressed among the $\mathrm{LN}+/ \mathrm{REC}+$, LN+/REC-, LN-/REC+, and LN-/REC- groups, eight of them also presented in the comparison of LN and REC clinical groups comparisons: miR-10a-5p, miR-1253, miR-1271-3p, miR-184, miR-18a-5p, miR411-5p, miR-542-3p, and again miR-595. Finally, a query of the KM Plot database of the TCGA and METABRIC TNBC cases, showed that several of the miRNAs observed with deregulated expression in the patients with larger tumor size, positive LN and REC were significantly associated with lower survival rates.

Interestingly, the involvement of 12 of the miRNAs observed in the TNBC cases of our study and that were differentially expressed in the above clinical groups was among the ones observed with differential expression in our previous study in TNBC cases of AA and non-Hispanic White (NHW) patients [24]. Among these common miRNAs, 12 of them presented the same level of expression of this study, two of which associated with tumor size (miR-2117 and miR-617), eight with LN status (miR-1253, miR-1268a, miR-200c-3p, miR-520d-5p, miR-518a-5p, miR-528, miR-580 and miR-873-5p), and two with REC status (miR-1200 and miR-449b-5p). These associations could indicate that these 12 miRNAs are intrinsically regulated in the TNBC of AA patients, and could account for the observed more aggressive phenotype of their tumors when compared to the NHW patients. This suggestion can be supported by the analysis of the KEGG pathway of the differentially expressed miRNAs observed in each clinical group of this study, which revealed their involvement in signaling pathways often associated with tumor aggressiveness. In the LN group, for example, considering only the panel of seven miRNAs that presented the best capability to discriminate $\mathrm{LN}+$ and $\mathrm{LN}$ - cases, among the top ten KEGG pathways observed, three were affected by six of these miRNAs: signaling pathways regulating pluripotency of stem cells, TGF-beta and Hippo signaling pathways. In the REC group, among the top ten KEGG pathways, the thyroid hormone signaling pathway was potentially affected by eight of the 12 miRNA in the highest discriminatory panel (miR-1200, miR-1271-3p, miR-449b-5p, miR-4536-3p, miR-542-3p, miR-548n, miR-593-3p and miR-595). Considering these miRNAs in the above clinical groups, miRNA/mRNA pairings that were experimentally validated showed a number of targets that play relevant roles in breast cancer progression, including genes that are part of the subclassification of the TNBC into the six molecular subtypes [68] among them, genes involved in the epithelial-to-mesenchymal transition (EMT) process, which is essential to confer cell 
migration and invasion and stem cell tumor capabilities [69, 70], and which miRNAs are important regulators $[71,72]$. Of note, one of the most prevalent TNBC subtypes in AA patients, the mesenchymallike (ML) subtype [73-75], is enriched in genes involved in the regulation of EMT and in the biology of cancer stem cells, both markers that confer clinical aggressiveness [76, 77]. In addition, some of the differentially expressed miRNAs observed in our study are highly involved in breast cancer progression as shown by their predicted interactions with relevant genes of the Integrated Breast Cancer Pathway. Some of these interactions were already experimentally validated, including miR-184 and AKT1K, BCL2 and MYC, and miR-200c, miR-130a-3p, miR-18a-5p, miR-519a-3p and PTEN. These interactions indicate an important role for these miRNAs in the tumorigenesis of TNBC in AA patients, which should be further explored in other independent well-characterized and large AA populations.

\section{Conclusion}

Altogether, our data indicate that alterations in miRNA expression are relevant biological factors that can be associated with the poor prognosis in TNBC of AA patients, by conferring to their TNBC cells, increase cell proliferation, elevate expression of angiogenesis markers and/or high migration and invasive cellular capabilities that are reflected in the clinical characteristics evaluated in this study. Therefore, apart from the socioeconomic and cultural factors that play a significant role in the observed disparities in incidence and mortality rates in TNBC of AA women, miRNAs deregulation can also be included as drivers of these disparities.

\section{Abbreviations}

AA: African-American; TNBC: triple negative breast cancer; LN: lymph node; REC: recurrence; ROC: Receiver Operating Characteristic; miRNAs: microRNAs; ER: estrogen receptor; PR: progesterone receptor; HER2: Human epidermal growth factor receptor-2; TCGA: The Cancer Genome Atlas; METABRIC: Molecular Taxonomy of Breast Cancer International Consortium; PPI: Protein-protein interactions; GO: biological process; HNSCC: head and neck squamous cell carcinoma; NSCLC: non-small cell lung cancer; EMT: Epithelial Mesenchymal Transition; NHW: non-Hispanic White; ML: mesenchymal-like.

\section{Declarations}

\section{Ethics approval and consent to participate}

All procedures and experimental protocols were approved by the Office of Regulatory Research Compliance - Institutional Review Board of Howard University (IRB-16-MED-39) and in accordance with the 1964 Helsinki declaration and its later amendments or comparable ethical standards. All the patients of this study signed informed consent for tissue procurement for research.

\section{Consent for publication}




\section{Availability of data and materials}

The datasets generated and analyzed during the current study are available in the "Data repository for Manuscript: A Panel of miRNAs as Prognostic Markers for African-American Patients with Triple Negative Breast Cancer" (https://app.box.com/s/9h5wfr95ixqjrcjvjm26ul5lg73w8kbv).

\section{Competing Interests}

The authors declare that all experiments comply with the current laws of the United States of America Conflict of Interest. The authors declare that they have no conflicts of interest.

\section{Funding}

This work was supported in part by the Charles \& Mary Latham Fund (Ella O. Latham Trust). The Genomics and Epigenomics Shared Resources (GESR) at Lombardi Comprehensive Cancer Center (LCCC) at Georgetown University was partially supported by the $\mathrm{NIH} / \mathrm{NCl}$ grant P30-CA051008. The Ohio State University Comprehensive Cancer Center (OSU CCC) was partially supported by the $\mathrm{NIH} / \mathrm{NCl}$ grant P30CA16058.

\section{Authors Contribution}

All authors made substantial contributions to one or more of the following: conceptualization and study design: LRC, YK; Data curation: ST, BMS, RLCJ, MC; formal Analysis: ST, BMS; funding acquisition: YK; methodology: ST, BMS, PF, RM, RLCJ, MC; resources: AA, TN, VA; supervision: LRC, YK; writing-original draft: ST, BMS; writing - review and editing: LRC, YK. All authors read and approved the final manuscript.

\section{Acknowledgments}

We thank the support of the Genomics and Epigenomics Shared Resources (GESR) at Lombardi Comprehensive Cancer Center (LCCC) at Georgetown University, partially supported by the $\mathrm{NIH} / \mathrm{NCl}$ grant P30-CA051008 for conducting the ancestral genomic analysis of the patients; and the Genomics Shared Resource at The Ohio State University Comprehensive Cancer Center (OSU CCC), supported in part by the OSU CCC and the $\mathrm{NIH} / \mathrm{NCl}$ grant P30-CA16058, for conducting the miRNA profiling hybridizations.

\section{References}


1. Siegel RL, Miller KD, Jemal A. Cancer statistics, 2020. CA Cancer J Clin. 2020; 70, 7-30. doi: 10.3322/caac. 21590 .

2. Kim M, Suh DH, Lee KH, Eom KY, Lee JY, Lee YY, et al. Major clinical research advances in gynecologic cancer in 2019. J Gynecol Oncol. 2020; 31, e48. doi: 10.3802/jgo.2019.30.e18.

3. Heimes AS, Schmidt M. Atezolizumab for the treatment of triple-negative breast cancer. Expert Opin Investig Drugs. 2019; 28, 1-5. doi: 10.1080/13543784.2019.1552255.

4. Marra A, Viale G, Curigliano G. Recent advances in triple negative breast cancer: The immunotherapy era. BMC Medicine. 2019; 17(1):90. doi:10.1186/s12916-019-1326-5.

5. Anders CK, Abramson V, Tan T, Dent R. The Evolution of Triple-Negative Breast Cancer: From Biology to Novel Therapeutics. Am Soc Clin Oncol Educ B. 2016; 36, 34-42. doi: 10.1200/EDBK_159135.

6. Hashemi A, Gorji-bahri G. MicroRNA: Promising Roles in Cancer Therapy. Curr Pharm Biotechnol. 2020; 21(12):1186-1203. doi:10.2174/1389201021666200420101613.

7. To KKW, Fong W, Tong CWS, Wu M, Yan W, Cho WCS. Advances in the discovery of microRNA-based anticancer therapeutics: latest tools and developments. Expert Opin Drug Discov. 2020; 15(1):63-83. doi:10.1080/17460441.2020.1690449.

8. Ramchandani D, Lee SK, Yomtoubian S, Han MS, Tung CH, Mittal V. Nanoparticle delivery of miR-708 mimetic impairs breast cancer metastasis. Mol Cancer Ther. 2019; 18(3):579-591. doi:10.1158/1535-7163.MCT-18-0702.

9. Yin H, Xiong G, Guo S, Xu C, Xu R, Guo P, et al. Delivery of Anti-miRNA for Triple-Negative Breast Cancer Therapy Using RNA Nanoparticles Targeting Stem Cell Marker CD133. Mol Ther. 2019; 27(7):1252-1261. doi:10.1016/j.ymthe.2019.04.018.

10. Ahir M, Upadhyay P, Ghosh A, Sarker S, Bhattacharya S, Gupta P, et al. Delivery of dual miRNA through CD44-targeted mesoporous silica nanoparticles for enhanced and effective triple-negative breast cancer therapy. Biomater Sci. 2020; 8(10):2939-2954. doi:10.1039/d0bm00015a.

11. O'Brien J, Hayder H, Zayed Y, Peng C. Overview of microRNA biogenesis, mechanisms of actions, and circulation. Front Endocrinol (Lausanne). 2018; 9, 1-12. doi: 10.3389/fendo.2018.00402.

12. Svoronos AA, Engelman DM, Slack FJ. OncomiR or Tumor Suppressor? The Duplicity of MicroRNAs in Cancer. Cancer Res. 2016; 76, 3666-3670. doi: 10.1158/0008-5472.CAN-16-0359.

13. Peng $Y$, Croce CM. The role of microRNAs in human cancer. Signal Transduct Target Ther. 2016; 1:15004. doi:10.1038/sigtrans.2015.4.

14. Van Roosbroeck K, Calin GA. Cancer Hallmarks and MicroRNAs: The Therapeutic Connection. Adv Cancer Res. 2017; 135:119-149. doi:10.1016/bs.acr.2017.06.002.

15. Teoh SL, Das S. The Role of MicroRNAs in Diagnosis, Prognosis, Metastasis and Resistant Cases in Breast Cancer. Curr Pharm Des. 2017; 23(12):1845-1859. doi:10.2174/1381612822666161027120043.

16. Kim J, Yao F, Xiao Z, Sun Y, Ma L. MicroRNAs and metastasis: small RNAs play big roles. 2018; Cancer Metastasis Rev. 37(1):5-15.doi:10.1007/s10555-017-9712-y. 
17. Vannini I, Fanini F, Fabbri M. Emerging roles of microRNAs in cancer. Curr Opin Genet Dev. 2018; 48:128-133. doi:10.1016/j.gde.2018.01.001.

18. Piasecka D, Braun M, Kordek R, Sadej R, Romanska H. MicroRNAs in regulation of triple-negative breast cancer progression. J Cancer Res Clin Oncol. 2018; 144, 1401-1411. doi: 10.1007/s00432018-2689-2.

19. Yao S, Graham K, Shen J, Campbell LES, Singh P, Zirpoli G, et al. Genetic variants in microRNAs and breast cancer risk in African American and European American women. Breast Cancer Res Treat. 2013; 141(3):447-59. doi:10.1007/s10549-013-2698-4.

20. Evans-Knowell A, LaRue AC, Findlay VJ. MicroRNAs and Their Impact on Breast Cancer, the Tumor Microenvironment, and Disparities. Adv Cancer Res. 2017; 133:51-76.

doi:10.1016/bs.acr.2016.08.003.

21. Bensen JT, Graff M, Young KL, Sethupathy P, Parker J, Pecot CV, et al. A survey of microRNA single nucleotide polymorphisms identifies novel breast cancer susceptibility loci in a case-control, population-based study of African-American women. Breast Cancer Res. 2018; 20(1):45. doi:10.1186/s13058-018-0964-4.

22. Telonis AG, Rigoutsos I. Race disparities in the contribution of miRNA isoforms and tRNA-derived fragments to Triple-negative breast cancer. Cancer Res. 2018; 78(5):1140-1154. doi:10.1158/00085472.CAN-17-1947.

23. Gong Z, Wang J, Wang D, Buas MF, Ren X, Freudenheim JL, et al. Differences in microRNA expression in breast cancer between women of African and European ancestry. Carcinogenesis. 2019; 40(1):6169. doi:10.1093/carcin/bgy 134 .

24. Sugita B, Gill M, Mahajan A, Duttargi A, Kirolikar S, Almeida R, et al. Differentially expressed miRNAs in triple negative breast cancer between African-American and non-Hispanic white women. Oncotarget. 2016; 7(48):79274-79291. doi:10.18632/oncotarget.13024.

25. Sugita BM, Pereira SR, de Almeida RC, Gill M, Mahajan A, Duttargi A, et al. Integrated copy number and miRNA expression analysis in triple negative breast cancer of Latin American patients. Oncotarget. 2019; 10(58):6184-6203. doi:10.18632/oncotarget.27250.

26. Hammond MEH, Hayes DF, Dowsett M, Allred DC, Hagerty KL, Badve S, et al. American society of clinical oncology/college of american pathologists guideline recommendations for immunohistochemical testing of estrogen and progesterone receptors in breast cancer. $\mathrm{J}$ Clin Oncol. 2010; 28(16):2784-95. doi:10.1200/JC0.2009.25.6529.

27. Wolff AC, Hammond MEH, Hicks DG, Dowsett M, McShane LM, Allison KH, et al. Recommendations for human epidermal growth factor receptor 2 testing in breast cancer: American society of clinical oncology/college of American pathologists clinical practice guideline update. Arch. Pathol Lab Med. 2014; 31(31):3997-4013. doi:10.5858/arpa.2013-0953-SA.

28. Yang J, Lee SH, Goddard ME, Visscher PM. GCTA: A tool for genome-wide complex trait analysis. Am J Hum Genet. 2011; 88(1):76-82. doi:10.1016/j.ajhg.2010.11.011 
29. Rstudio Team. RStudio: Integrated development for R. RStudio, Inc., Boston MA. RStudio. 2019. http://www.rstudio.com/

30. Zakharia F, Basu A, Absher D, Assimes TL, Go AS, Hlatky MA, et al. Characterizing the admixed African ancestry of African Americans. Genome Biol. 2099; doi:10.1186/gb-2009-10-12-r141.

31. Petersen DC, Libiger O, Tindall EA, Hardie RA, Hannick LI, Glashoff RH, et al. Complex Patterns of Genomic Admixture within Southern Africa. PLoS Genet. 2013;9(3):e1003309. doi:10.1371/journal.pgen.1003309.

32. Nagy A, Lánczky A, Menyhárt 0 , Győrffy B. Validation of miRNA prognostic power in hepatocellular carcinoma using expression data of independent datasets. Sci Rep. 2018; 15;8(1):9227. doi: 10.1038/s41598-018-27521-y.

33. Vlachos IS, Zagganas K, Paraskevopoulou MD, Georgakilas G, Karagkouni D, Vergoulis T, et al. DIANA-miRPath v3.0: Deciphering microRNA function with experimental support. Nucleic Acids Res. 2015; 43(W1):W460-6. doi:10.1093/nar/gkv403.

34. Kehl T, Kern F, Backes C, Fehlmann T, Stöckel D, Meese E, et al. miRPathDB 2.0: a novel release of the miRNA Pathway Dictionary Database. Nucleic Acids Res. 2020; 8;48(D1):D142-D147. doi: 10.1093/nar/gkz1022.

35. Slenter DN, Kutmon M, Hanspers K, Riutta A, Windsor J, Nunes N, et al. WikiPathways: a multifaceted pathway database bridging metabolomics to other omics research. Nucleic Acids Res. 2018; 4;46(D1):D661-D667. doi: 10.1093/nar/gkx1064.

36. Huang H, Wu X, Ibrahim S, McKenzie M, Chen JY. Predicting Drug Efficacy Based on the Integrated Breast Cancer Pathway Model. IEEE. 2011; 42-45. doi: 10.1109/GENSiPS.2011.6169437.

37. Kahraman M, Röske A, Laufer T, Fehlmann T, Backes $C$, Kern F, et al. MicroRNA in diagnosis and therapy monitoring of early-stage triple-negative breast cancer. Sci Rep. 2018; 8(1):11584. doi:10.1038/s41598-018-29917-2.

38. Chou CH, Shrestha S, Yang CD, Chang NW, Lin YL, Liao KW, et al. MiRTarBase update 2018: A resource for experimentally validated microRNA-target interactions. Nucleic Acids Res. 2018; 46(D1):D296-D302. doi:10.1093/nar/gkx1067.

39. Fan Y, Xia J. miRNet-Functional analysis and visual exploration of miRNA-target interactions in a network context. Methods Mol Biol. 2018; 1819:215-233. doi:10.1007/978-1-4939-8618-7_10.

40. Hamberg M, Backes C, Fehlmann T, Hart M, Meder B, Meese E, Keller A. miRTargetLink-miRNAs, Genes and Interaction Networks. Int J Mol Sci. 2016; 17(4). doi: http://doi.org/10.3390/ijms17040564.

41. Szklarczyk D, Gable AL, Lyon D, Junge A, Wyder S, Huerta-Cepas J, et al. STRING v11: protein-protein association networks with increased coverage, supporting functional discovery in genome-wide experimental datasets. Nucleic Acids Res. 2019; 47:D607-613. doi: 10.1093/nar/gky1131.

42. Shannon P, Markiel A, Ozier O, Baliga NS, Wang JT, Ramage D, et al. Cytoscape: a software environment for integrated models of biomolecular interaction networks. Genome Res. 2003; 13(11):2498-504. doi: 10.1101/gr.1239303.

Page 23/30 
43. Fan C, Liu N. Identification of dysregulated microRNAs associated with diagnosis and prognosis in triple-negative breast cancer: An in silico study. Oncol Rep. 2019; 41(6):3313-3324. doi:10.3892/or.2019.7094.

44. Tang Q, Ouyang H, He D, Yu C, Tang G. MicroRNA-based potential diagnostic, prognostic and therapeutic applications in triple-negative breast cancer. Artif Cells Nanomed Biotechnol. 2019; 47(1):2800-2809. doi:10.1080/21691401.2019.1638791.

45. Carter CL, Allen C, Henson DE. Relation of tumor size, lymph node status, and survival in 24,740 breast cancer cases. Cancer. 1989; 63(1):181-7. doi:10.1002/1097-0142(19890101)63:1<181::AIDNCR2820630129>3.0.CO;2-H.

46. Elkin EB, Hudis C, Begg CB, Schrag D. The effect of changes in tumor size on breast carcinoma survival in the U.S.: 1975-1999. Cancer. 2005; 104(6):1149-57. doi:10.1002/cncr.21285.

47. Slattery ML, Mullany LE, Sakoda LC, Wolff RK, Samowitz WS, Herrick JS. The MAPK-Signaling Pathway in Colorectal Cancer: Dysregulated Genes and Their Association With MicroRNAs. Cancer Inform. 2018; 26;17. doi: 10.1177/1176935118766522.

48. Pasche B, Pennison MJ, Jimenez H, Wang M. TGFBR1 and Cancer Susceptibility. Trans Am Clin Climatol Assoc. 2014; 125: 300-312.

49. Lopez YON, Victoria B, Golusinski P, Golusinski W, Masternak MM. Characteristic miRNA expression signature and random forest survival analysis identify potential cancer-driving miRNAs in a broad range of head and neck squamous cell carcinoma subtypes. Rep Pract Oncol Radiother. 2018; 23(1): 6-20. doi: 10.1016/j.rpor.2017.10.003.

50. Wang L, Mo H, Jiang Y, Wang Y, Sun L, Yao B, et al. MicroRNA-519c-3p promotes tumor growth and metastasis of hepatocellular carcinoma by targeting BTG3. Biomed Pharmacother. 2019; 118:109267. doi: 10.1016/j.biopha.2019.109267.

51. Castilla MA, López-García MA, Atienza MR, Rosa-Rosa JM, Díaz-Martín J, Pecero ML, et al. VGLL1 expression is associated with a triple-negative basal-like phenotype in breast cancer. Endocr Relat Cancer. 2014; 21(4):587-99. doi: 10.1530/ERC-13-0485.

52. Zahoor S, Haji A, Battoo A, Qurieshi M, Mir W, Shah M et al. Sentinel lymph node biopsy in breast cancer: A clinical review and update. J Breast Cancer. 2017; 20(3):217-227. doi:10.4048/jbc.2017.20.3.217.

53. Liu C, Yang H, Xu Z, Li D, Zhou M, Xiao K, et al. microRNA-548I is involved in the migration and invasion of non-small cell lung cancer by targeting the AKT1 signaling pathway. J Cancer Res Clin Oncol. 2015; 141(3):431-41. doi: 10.1007/s00432-014-1836-7.

54. Liu M, Zhang Y, Zhang J, Cai H, Zhang C, Yang Z, et al. MicroRNA-1253 suppresses cell proliferation and invasion of non-small-cell lung carcinoma by targeting WNT5A. Cell Death Dis. 2018; 7;9(2):189. doi: 10.1038/s41419-017-0218-x.

55. Tang L, Chen Y, Tang X, Wei D, Xu X, Yan F. Long Noncoding RNA DCST1-AS1 Promotes Cell Proliferation and Metastasis in Triple-negative Breast Cancer by Forming a Positive Regulatory Loop with miR-873-5p and MYC. J Cancer. 2020; 11(2):311-323. doi:10.7150/jca.33982. 
56. Ding J, Wu W, Yang J, Wu M. Long non-coding RNA MIF-AS1 promotes breast cancer cell proliferation, migration and EMT process through regulating miR-1249-3p/HOXB8 axis. Pathol Res Pract. 2019; 215(7):152376. doi: 10.1016/j.prp.2019.03.005.

57. Pan B, Zhao M, Wang N, Xu L, Wu T, Li Z. LncRNA RGMB-AS1 Promotes Glioma Growth and Invasion Through miR-1200/HOXB2 Axis. Onco Targets Ther. 2019; 12:10107-10114. doi: 10.2147/OTT.S230098.

58. Li S, Pei Y, Wang W, Liu F, Zheng K, Zhang X. Circular RNA 0001785 regulates the pathogenesis of osteosarcoma as a ceRNA by sponging miR-1200 to upregulate HOXB2. Cell Cycle. 2019; 18(11):1281-1291. doi: 10.1080/15384101.2019.1618127.

59. Loibl S. Neoadjuvant treatment of breast cancer: Maximizing pathologic complete response rates to improve prognosis. Curr Opin Obstet Gynecol. 2015; 27(1):85-91. doi:10.1097/GCO.0000000000000147.

60. Harbeck N, Gluz O. Neoadjuvant therapy for triple negative and HER2-positive early breast cancer. Breast. 2017; S99-S103. doi:10.1016/j.breast.2017.06.038.

61. Lebert JM, Lester R, Powell E, Seal M, McCarthy J. Advances in the systemic treatment of triplenegative breast cancer. Curr Oncol. 2018; S142-S150. doi:10.3747/co.25.3954.

62. Carey LA, Dees EC, Sawyer L, Gatti L, Moore DT, Collichio F, et al. The triple negative paradox: Primary tumor chemosensitivity of breast cancer subtypes. Clin Cancer Res. 2007; 2329-34. doi:10.1158/1078-0432.CCR-06-1109.

63. Sheppard VB, Isaacs C, Luta G, Willey SC, Boisvert M, Harper FWK, et al. Narrowing racial gaps in breast cancer chemotherapy initiation: The role of the patient-provider relationship. Breast Cancer Res Treat. 2013; 139(1):207-16. doi:10.1007/s10549-013-2520-3.

64. Killelea BK, Yang VQ, Wang SY, Hayse B, Mougalian S, Horowitz NR, et al. Racial differences in the use and outcome of neoadjuvant chemotherapy for breast cancer: Results From the National Cancer Data Base. J Clin Oncol. 2015; 33(36):4267-76. doi:10.1200/JC0.2015.63.7801.

65. Yee MK, Sereika SM, Bender CM, Brufsky AM, Connolly MC, Rosenzweig MQ. Symptom incidence, distress, cancer-related distress, and adherence to chemotherapy among African American women with breast cancer. Cancer. 2017; 123(11):2061-2069. doi:10.1002/cncr.30575.

66. Gallups SF, Connolly MC, Bender CM, Rosenzweig MQ. Predictors of Adherence and Treatment Delays among African American Women Recommended to Receive Breast Cancer Chemotherapy. Women's Health Issues. 2018; 28(6):553-558. doi:10.1016/j.whi.2018.08.001.

67. Sheppard VB, Cavalli LR, Dash C, Kanaan YM, Dilawari AA, Horton S, et al. Correlates of Triple Negative Breast Cancer and Chemotherapy Patterns in Black and White Women With Breast Cancer. Clin Breast Cancer. 2017; 17(3):232-238. doi:10.1016/j.clbc.2016.12.006.

68. Lehmann BD, Bauer JA, Chen X, Sanders ME, Chakravarthy AB, Shyr Y, et al. Identification of human triple-negative breast cancer subtypes and preclinical models for selection of targeted therapies. $J$ Clin Invest. 2011; 121(7):2750-67. doi:10.1172/JCI45014. 
69. Lim J, Thiery JP. Epithelial-mesenchymal transitions: Insights from development. Development. 2012; 139(19):3471-86. doi:10.1242/dev.071209.

70. Craene BDe, Berx G. Regulatory networks defining EMT during cancer initiation and progression. Nat Rev Cancer. 2013; 13(2):97-110. doi:10.1038/nrc3447.

71. Tang J, Li Y, Wang J, Wen Z, Lai M, Zhang H. Molecular mechanisms of microRNAs in regulating epithelial-mesenchymal transitions in human cancers. Cancer Letters. 2016; 371(2):301-13. doi:10.1016/j.canlet.2015.11.043.

72. Zhao X, Lu Y, Nie Y, Fan D. MicroRNAs as Critical Regulators Involved in Regulating EpithelialMesenchymal Transition. Curr Cancer Drug Targets. 2014; 13(9):935-44. doi:10.2174/15680096113136660099.

73. Field LA, Love B, Deyarmin B, Hooke JA, Shriver CD, Ellsworth RE. Identification of differentially expressed genes in breast tumors from African American compared with Caucasian women. Cancer. 2012; 118(5):1334-44. doi:10.1002/cncr.26405.

74. Lindner R, Sullivan C, Offor O, Lezon-Geyda K, Halligan K, Fischbach N, et al. Molecular phenotypes in triple negative breast cancer from African American patients suggest targets for therapy. PLoS One. 2013; 8(11):e71915. doi:10.1371/journal.pone.0071915.

75. Stewart PA, Luks J, Roycik MD, Sang QXA, Zhang J. Differentially expressed transcripts and dysregulated signaling pathways and networks in African American breast cancer. PLoS One. 2013; 8(12):e82460. doi:10.1371/journal.pone.0082460.

76. Wang SS, Jiang J, Liang XH, Tang YL. Links between cancer stem cells and epithelialmesenchymal transition. Onco Targets Ther. 2015; 8:2973-80. doi:10.2147/OTT.S91863.

77. Liu X, Fan D. The Epithelial-Mesenchymal Transition and Cancer Stem Cells: Functional and Mechanistic Links Curr Pharm Des. 2015; 21(10):1279-91. doi:10.2174/1381612821666141211115611.

\section{Figures}

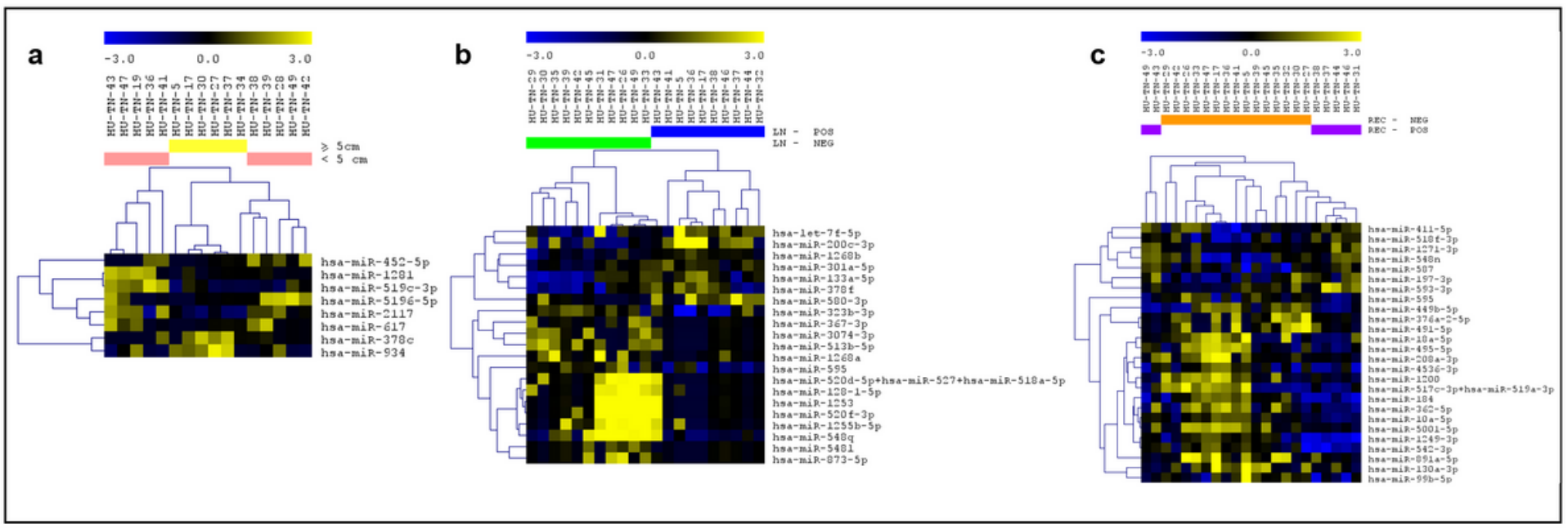


Figure 1

Differentially expressed miRNAs in the analysis of the TNBC cases distributed by tumor size (a), LN (b) and REC (c) status.

\section{$\begin{array}{lll}-3.0 & 0.0 & 3.0\end{array}$

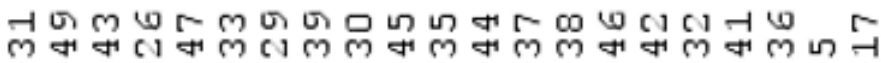

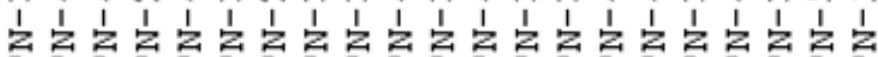

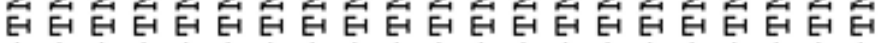

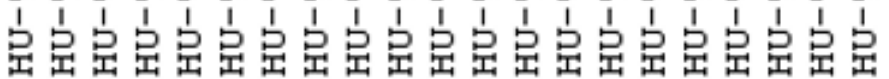
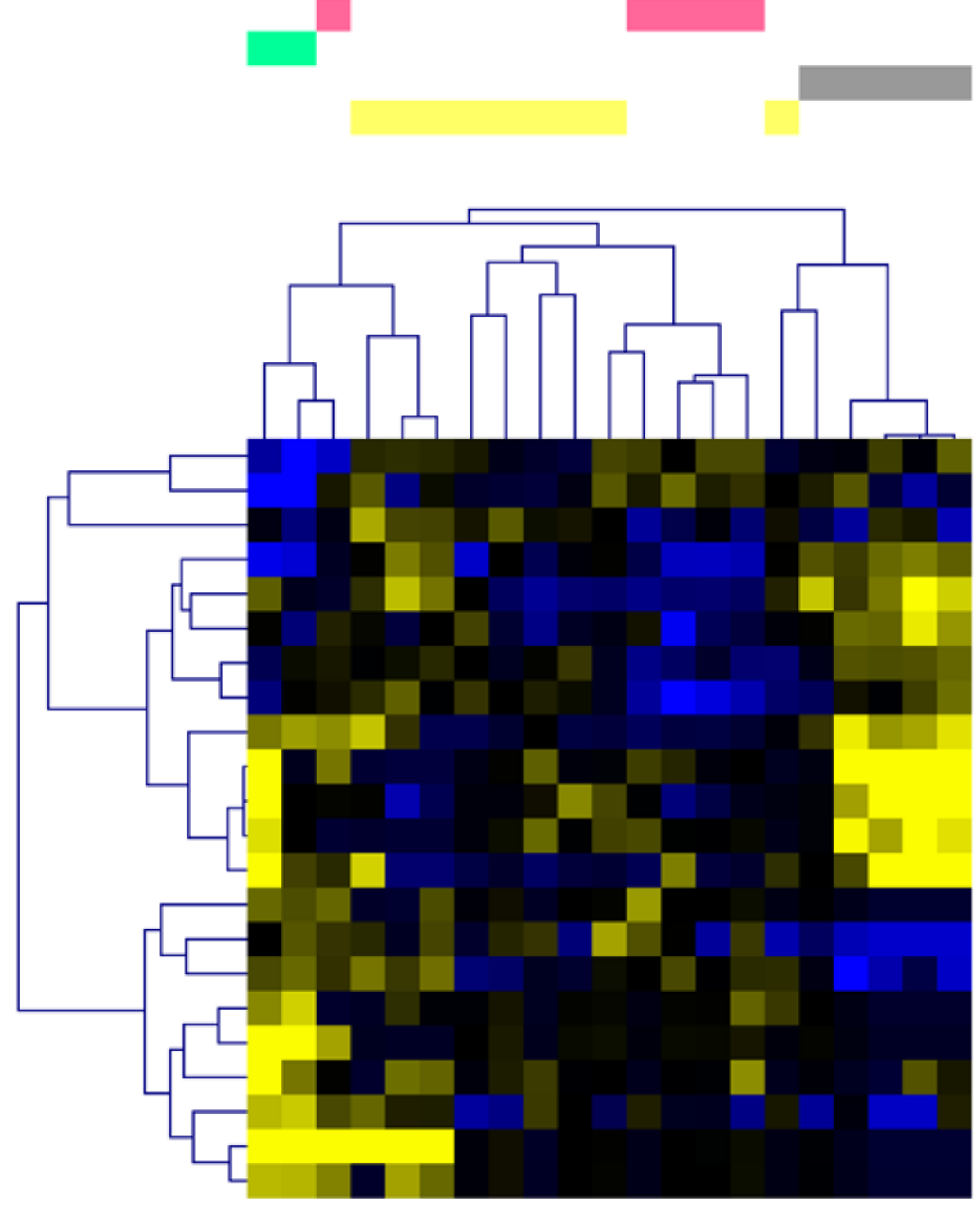

$\mathrm{LN}+/ \mathrm{REC}+$

$\mathrm{LN}-/ \mathrm{REC}+$

$\mathrm{LN}+/ \mathrm{REC}-$

$\mathrm{LN}-/ \mathrm{REC}-$

hsa-miR-1295a

hsa-miR-520c-3p

hsa-miR-595

hsa-miR-184

hsa-miR-18a-5p

hsa-miR-34a-5p

hsa-miR-10a-5p

hsa-miR-542-3p

hsa-miR-548m

hsa-miR-126-3p

hsa-miR-25-3p

hsa-miR-30d-5p

hsa-miR-93-5p

hsa-miR-1271-3p

hsa-miR-216b-5p

hsa-miR-411-5p

hs a-miR-219a-1-3p

hsa-miR-502-5p

hsa-miR-92a-3p

hsa-miR-4536-5p

hsa-miR-1253

hsa-miR-944

Figure 2 
Differentially expressed miRNAs in the analysis of the TNBC cases distributed in the four combined LN and REC status groups.

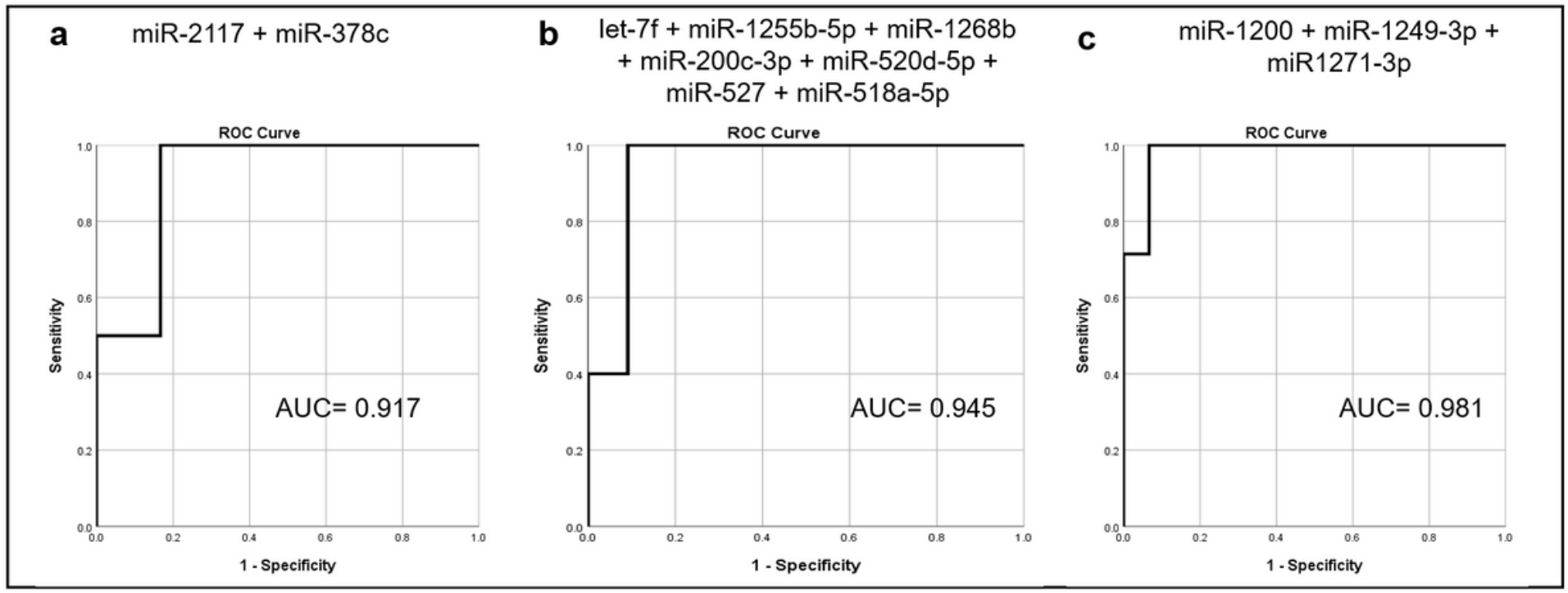

Figure 3

Combined ROC analysis for the most robust discriminatory miRNA panels for tumor size (a), LN (b) and REC (c) status.

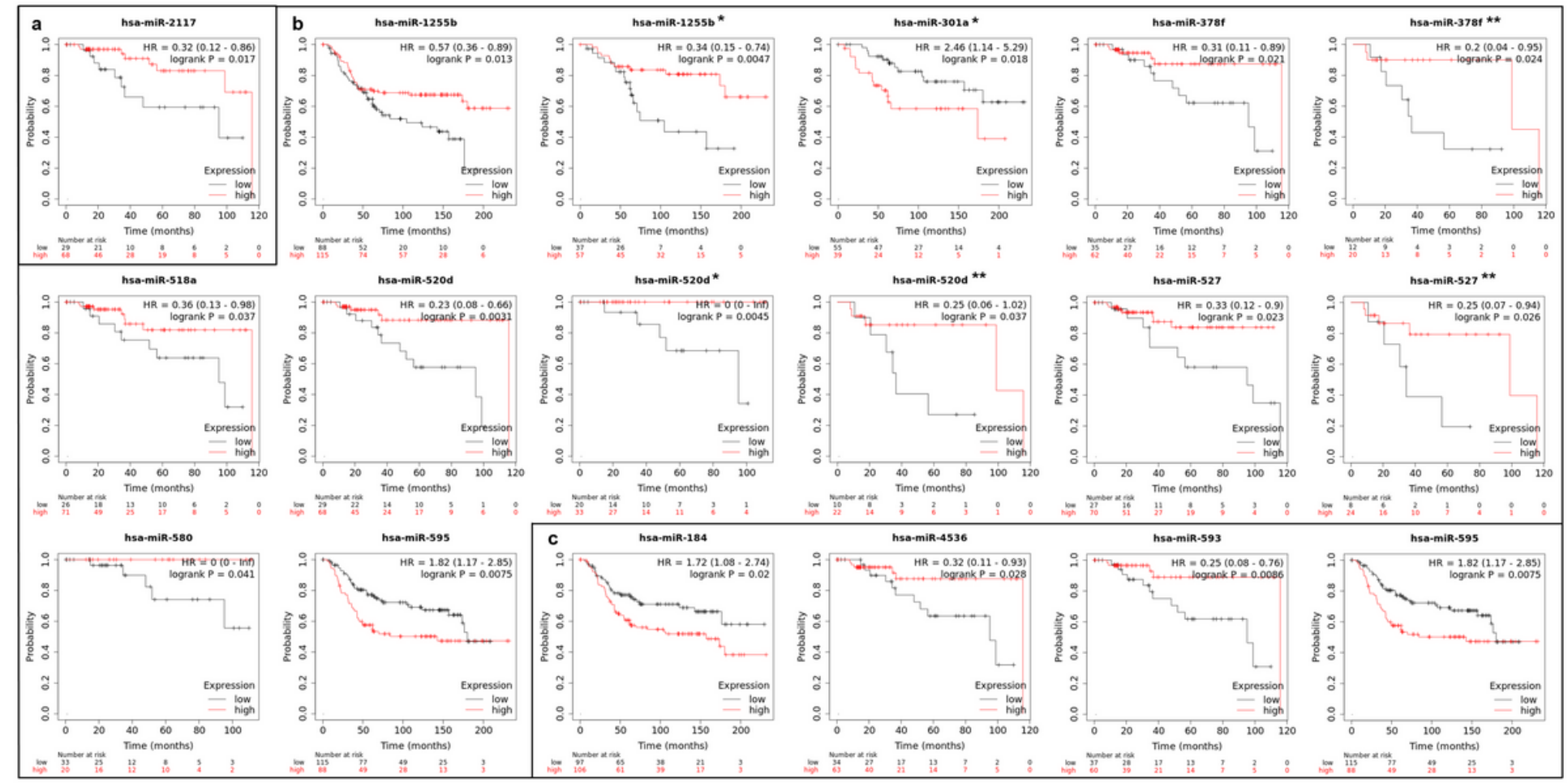

\section{Figure 4}

KM Plot graphics of the miRNAs that presented AUC $>0.8$ in each clinical group analyzed (a: tumor size, b:LN, and c: REC) and that were significantly associated with survival in the TNBC cases of TCGA and/or METABRIC. * indicates patients with $\mathrm{LN}$ - and ** with $\mathrm{LN}+$. 
a

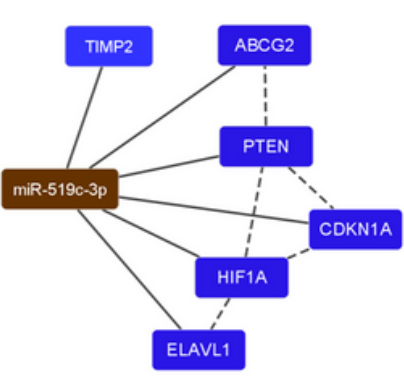

C

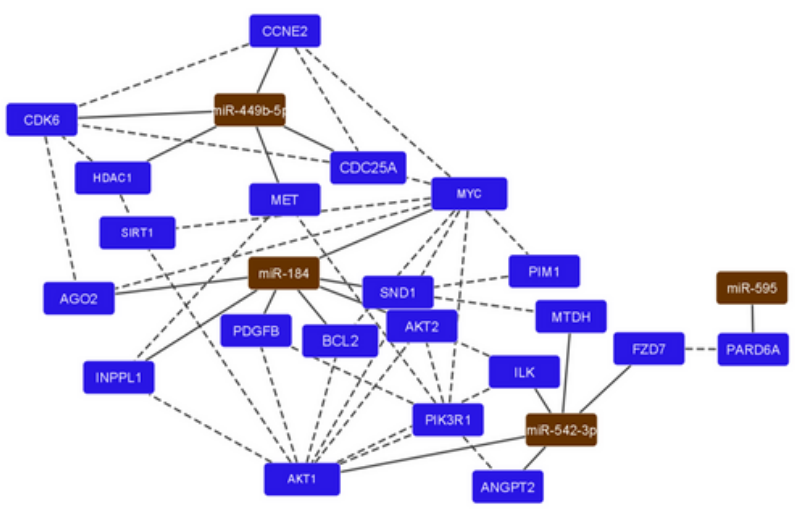

b

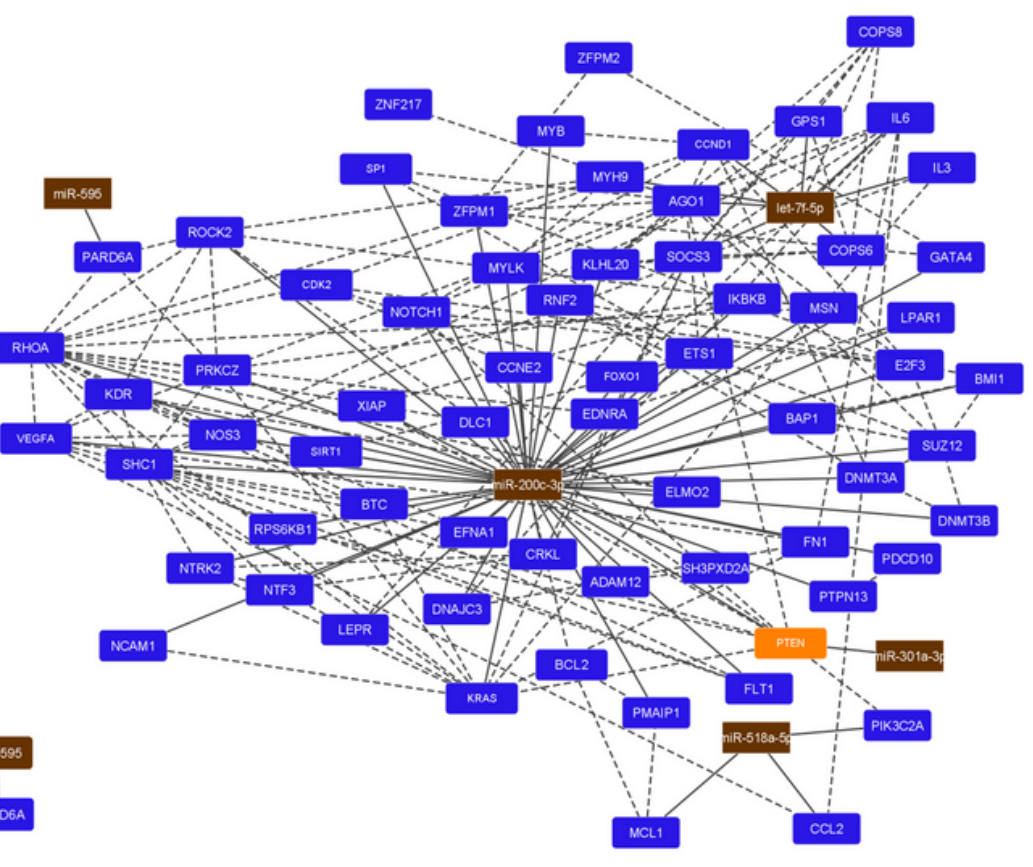

Figure 5

MiRNAmRNA networks of the differentially expressed miRNAs observed in the tumor size (a), LN (b) an REC (c) clinical groups and their corresponding experimentally validated target genes (Cytoscape v. 3.8.0).

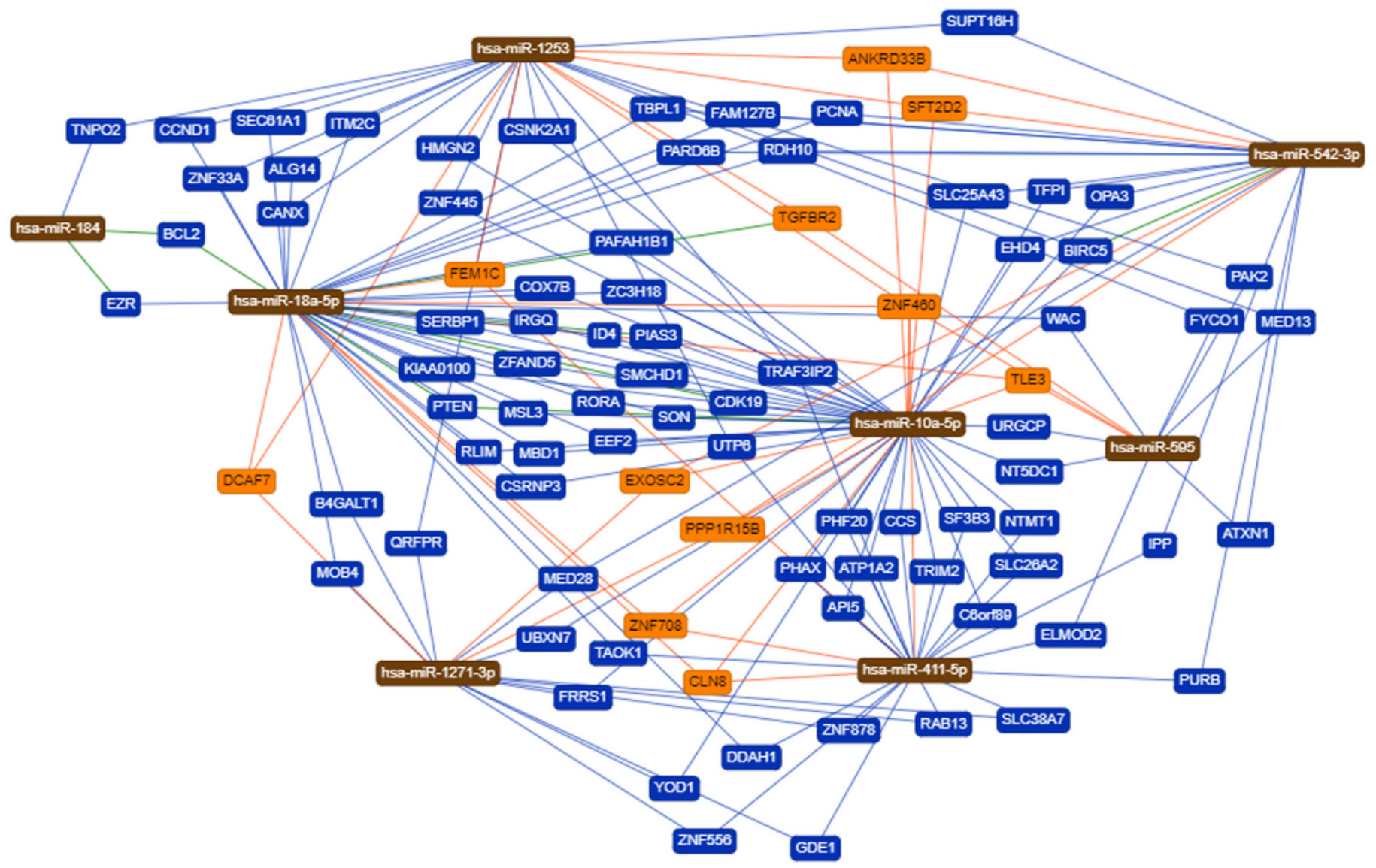


Figure 6

MiRNA/mRNA network of the 8 miRNAs commonly observed in the to LN/REC, LN and REC groups' comparisons (miRTargetLink)

\section{Supplementary Files}

This is a list of supplementary files associated with this preprint. Click to download.

- TurkistanietalFigureS1.tif

- TurkistanietalFigureS2.tif

- TurkistanietalTableS1.docx

- TurkistanietalTableS2.docx

- TurkistanietalTableS3.docx 